\title{
An intraoral device for weight loss: initial clinical findings
}

\author{
Paul A. Brunton, ${ }^{* 1}$ Jithendra Ratnayake, ${ }^{1}$ H. Jonathan Bodansky, ${ }^{2}$ Li Mei, ${ }^{1}$ Arthi Veerasamy ${ }^{1}$ and Richard Hall ${ }^{3}$
}

\section{Key points}

Obesity rates have increased at an alarming rate and nearly a third of the world's population is now classified as overweight or obese.
This study investigated the acceptability and tolerability of a novel intra-oral device to facilitate weight loss in obese patients.
Tolerance of the device was good, promoting weight loss during a two-week trial period.

\begin{abstract}
Introduction Obesity is a global epidemic, increasing the risk of many associated health issues.

Aim The aim of this clinical study was to investigate the acceptability and tolerability of an intraoral device, designed to facilitate weight loss.
\end{abstract}

Method Seven healthy obese participants were recruited. The device, which incorporated closed-field magnets with keepers to restrict mouth opening, was cemented to the participants' first molars. The participants were given a commercially available liquid diet for two weeks. The comfort and tolerability of the device were assessed using a quality of life questionnaire during review appointments at 1, 7 and 14 days and two weeks after device removal.

Results The participants reached a mean weight loss of 6.36 (SD = 3.79) kilograms, which represents approximately $5.1 \%$ of their body weight. The participants had trouble pronouncing some words and felt tense and embarrassed 'only occasionally'. The participants 'hardly ever' reported a change in taste sensation or felt uncomfortable drinking. However, participants indicated that they occasionally had discomfort and felt that life in general was less satisfying. Qualitative analysis showed that the participants were happy with the outcome and were motivated to lose more weight.

Conclusion The participants tolerated the device for a two-week period with satisfactory weight loss and were further motivated to continue their weight loss journey.

\section{Introduction}

Obesity is increasingly common, with a global epidemic in both developed and developing countries now being reported. ${ }^{1}$ Recent studies have reported that 1.9 billion adults are overweight and 650 million are obese, with approximately 2.8 million deaths reported annually as a result of being overweight or obese. ${ }^{2}$ Moreover, by 2030, it was estimated that $57.8 \%$ of the adult population could be either overweight or obese. ${ }^{3}$ Obesity is defined as having an excessively high amount of body fat (adipose tissue) in relation to lean

${ }^{1}$ University of Otago, Faculty of Dentistry, 310 Great King Street, North Dunedin 9016, New Zealand; '2Leeds Teaching Hospitals NHS Trust, Leeds, UK; ${ }^{3}$ RMH Consultancy, Leeds, UK.

${ }^{\star}$ Correspondence to: Paul Brunton

Email address: paul.brunton@otago.ac.nz

Refereed Paper

Accepted 24 September 2020

https://doi.org/10.1038/s41415-021-3081-1 body mass and is associated with several health risks including diabetes, hypertension, cardiovascular disease, respiratory dysfunction, gallstone disease, arthritis, reduced mobility and certain forms of cancer. ${ }^{4,5}$ In addition, psychological symptoms may be present including embarrassment, depression and loss of self-esteem, and obese people may suffer eating disorders, together with stigmatisation and discrimination. ${ }^{6}$

At present, the world is reeling from the health and social impact of the global pandemic of COVID-19, and people with obesity are at increased risk of severe complications if infected with COVID-19 and requiring subsequent hospital admission and invasive ventilation. ${ }^{7,8}$ Weight reduction may improve life expectancy and also reverse many of the medical conditions that are associated with obesity. ${ }^{9,10}$ Alterations in diet and physical activity are the mainstays of initial treatment. Calorie restriction and increased energy expenditure should be successful, although in many people the effects are either negligible or only successful in the short term because of poor motivation and brief adherence to lifestyle changes. ${ }^{10,11}$ A variety of drugs have been tried as adjuncts to diet therapy. ${ }^{12}$ Earlier drugs were related to the amphetamine group and had serious side effects, some addictive properties and are not generally used today. More recent derivatives of those drugs have been withdrawn from the market because of potential serious side effects. ${ }^{13}$ Drug therapy has only been proven to be helpful in the short or medium term, rather than in the long term, and continued drug therapy may not only have side effects but is also expensive. However, once morbid obesity is present, eating habits are firmly established and difficult to change in an environment of plentiful food, exercise is limited by body bulk, and drugs and diet will have little effect. As a result, bariatric surgery has become an increasingly popular treatment choice for patients who have not succeeded with lifestyle changes or medical management. 
Although bariatric surgery results in superior outcomes in terms of weight loss, it is invasive, irreversible, costly and may not suit every patient, particularly those who are frail or who decline surgery due to potential risks. ${ }^{14,15,16}$ In the 1980s, jaw wiring (maxillomandibular fixation) was tried as an aid to weight reduction, mainly by dentists and physicians. ${ }^{17,18}$ After jaw wiring, only fluids could be taken and weight losses of 20-30 kilograms $(\mathrm{kg})$ in six months were common. ${ }^{18,19}$ However, many jaw-wired patients felt anxious and some developed acute psychiatric conditions. ${ }^{18}$ In addition, after 9-12 months, patients developed periodontal disease. After wire removal, a transient, and in some cases persistent, limitation of jaw movement was also observed. ${ }^{18,20}$

Although metabolic/bariatric surgery plays a major role in the management of the morbidly obese today, it cannot be relied upon to manage the global obesity epidemic. Alternative strategies are required which may obviate surgery, or which reduce weight before surgery and so make it easier and safer. A device which contains magnets provides a novel approach to the treatment of obesity and can be used in the short, medium or long term, either continuously or intermittently. The main barrier to successful weight loss with dietary advice and restriction is poor patient adherence. The device aids adherence for the majority of those whose dietary adherence is very short-term and needs physical adjunctive treatment. Furthermore, magnetic devices offer a realistic, attractive and economic alternative to surgical procedures. With this in mind, the aim of this study is to investigate the practicality, comfort, tolerability and safety of an intraoral device and also to determine the magnitude of weight loss achieved during the two-week study period.

\section{Materials and method}

Ethical approval was obtained from the Health and Disability Ethics Committees (HDEC) (approval number 16/NTB/89), and the study was conducted in full accordance with the World Medical Declaration of Helsinki and conformed to the STROBE statement for observational studies. The clinical trial was registered in the Australia New Zealand Clinical Trials Registry (https://www.anzctr.org.au) and received the registration ID ACTRN12616001198415. This study recruited 28 obese volunteer participants with a Body Mass Index (BMI) $>35$ from the wider community in Dunedin, New Zealand.

\begin{tabular}{l|l|l}
\multicolumn{2}{|c}{ Table 1 Inclusion and exclusion criteria for participant selection } \\
\hline Number & Inclusion criteria & Exclusion criteria \\
\hline 1 & BMI $>35$ & $\begin{array}{l}\text { Participants using continuous positive airway } \\
\text { pressure devices to manage sleep apnoea }\end{array}$ \\
\hline 2 & $\begin{array}{l}\text { No major co-occurring health conditions (eg } \\
\text { asthma, heart problems) }\end{array}$ & $\begin{array}{l}\text { Diabetic patients on insulin and oral } \\
\text { hypoglycaemic therapy }\end{array}$ \\
\hline 3 & $\begin{array}{l}\text { A healthy mouth, free of periodontal disease } \\
\text { and dental caries, with a sound posterior } \\
\text { dentition with no missing teeth }\end{array}$ & $\begin{array}{l}\text { Participants who were on oral medication that } \\
\text { cannot be given in liquid form }\end{array}$ \\
\hline
\end{tabular}

The inclusion and exclusion criteria for the participants are summarised in Table 1. Seven of the volunteer participants met the inclusion criteria.

After obtaining written, informed consent, the participants provided demographic information to include their name, contact information, date of birth, sex and self-reported ethnicity. A dental inspection was conducted by the primary investigator to confirm each individual's suitability for the study. Baseline screening tests were performed including full dental charting, Community Periodontal Index of Treatment Needs (CPITN), medical history, dietary assessment, and measurement of height and weight.

Seven participants of those screened met the inclusion criteria. Elastic separators (AlastiK, $3 \mathrm{M}$, New Zealand) which are commonly used in orthodontics were placed on the first molars to allow tooth separation. When adequate separation of the teeth was achieved, a dental impression (Polyether, Impregum, 3M, New Zealand) was taken to produce a working model on which the dental device would be manufactured. The dental device, used in this study, consisted of stainless steel orthodontictype metal bands (NC Plain Molar Bands, 3M, USA) fixed to the upper and lower posterior teeth by a combination of a glass-ionomerbased orthodontic cement (Transbond XT adhesive, 3M, New Zealand) and unique, custom-manufactured locking bolts (Fig. 1).

Closed-field magnets which had an internal

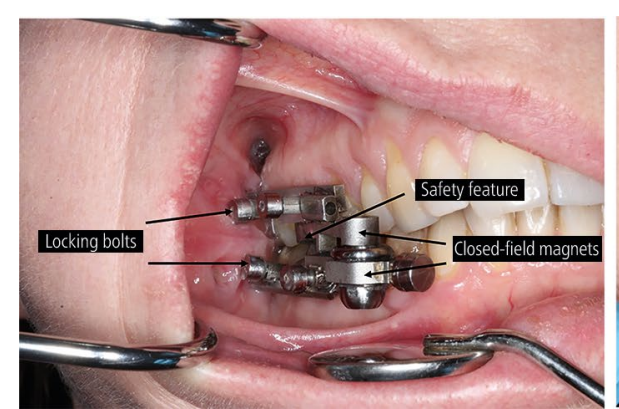

Fig. 1 Intraoral device consisting of closed-field magnets and orthodontic bands
O-ring element (MBI-Technovent Limited, UK) together with keepers provided the mechanical resistance which restricted mouth opening. The resistance was modified using a silicone O-ring (MBI-Technovent Limited, UK). However, the design, which consisted of magnetic and mechanical attachments, allowed some movement of the temporomandibular joint so that discomfort and stiffness were avoided. ${ }^{21}$ The device also consisted of siliconebased cheek protectors (Techsil-20 HCR MBITechnovent Limited, UK), which prevented any friction against the cheeks.

A safety feature was incorporated into the device which allowed for the disengagement of the device in the case of an emergency, such as a panic attack or where a possibility of choking arises. This device was demonstrated to the subject and another individual of their choosing and they were asked to keep the emergency release device with them at all times. The device was designed such that the configuration maintained the airway, allowed speech and allowed feeding using a liquid diet.

With the device in place, the participants were given careful instruction with respect to maintaining oral hygiene, and a mouthwash was dispensed (Colgate Savacol, Colgate-Palmolive, Auckland, New Zealand) and a commercially available liquid diet, Fortisip Drinks (Nutricia, New Zealand), for two weeks. The diet supplied $300 \mathrm{kcal}$ of energy in a $200 \mathrm{ml}$ bottle. The participants were prescribed $800 \mathrm{ml}(1,200 \mathrm{kcal})$ per day in the form of four drinks, plus one

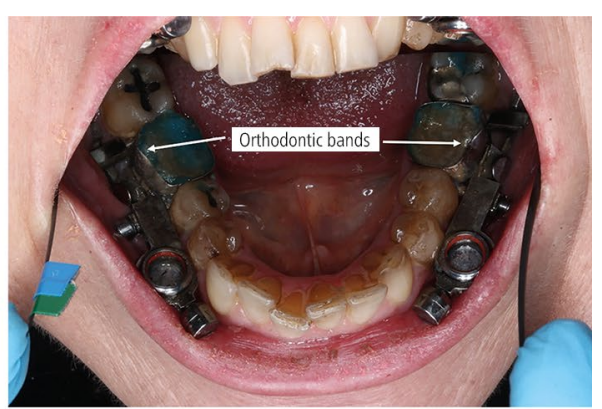

\section{(1)}


protein shake (25 g of protein; Horleys, New Zealand) and unrestricted low-calorie liquids such as tea and coffee. During the two-week study period, the participants received dental, dietetic and medical supervision. The comfort and tolerability of the device were measured using a self-administered questionnaire using a Likert scale $(1=$ never; 2 = hardly ever; 3 = occasionally; 4 = fairly often; 5 = very often). The quality of life (QoL) was measured using an impact of weight and QoL during review appointments at baseline, 1,7 and 14 days, and 14 days post-device removal. ${ }^{22,23}$ The participants ranked their QoL using a Likert scale questionnaire $(5=$ never true; 4 = rarely true; 3 = sometimes true; 2 = usually true; 1 = always true).

After 14 days, the device was removed and the occlusion was checked to confirm there had been no changes, and this was done based on patient feedback. Periodontal health was confirmed by confirming an absence or not of bleeding on probing. Patients were advised to contact the research team if they had any cause for concern.

\section{Results}

Six participants completed the study and one patient left the study after eight days for reasons unrelated to the study. One patient who completed the study was unable to attend the review appointment 14 days after device removal after being diagnosed with acute rheumatoid arthritis requiring hospital admission. No changes to the subject's occlusion were noted when the device was removed. In addition, no deterioration in periodontal health was noted apart from some bleeding on probing on the lingual and palatal aspects of the teeth used to retain the device, which resolved once traditional plaque control measures were reintroduced. At no point did any of the subjects feel the need to use the emergency release device.

\section{Demographics}

Of the seven participants, all were women and were of European ethnicity. The mean age of the participants was 36.71 years, and the mean height and weight of the participants was $162.5 \mathrm{~cm}( \pm 5.59)$ and $107.98 \mathrm{~kg}( \pm 23.22)$, respectively. The mean BMI of the participants at baseline was $40.84( \pm 8.27)$. Of the seven participants who took part in the study, all lost weight, with a mean weight loss of 6.36 $\mathrm{kg}(\mathrm{SD}=3.79)$ of their body weight. However, two weeks following device removal, all the participants gained some weight, with an average weight gain of approximately $0.73 \mathrm{~kg}$ (Fig. 2).

\section{Device tolerability}

Table 2 shows the device tolerability of the intraoral device. The tolerability was assessed using a modified device tolerability questionnaire. The participants were asked to fill out the questionnaire during baseline (before placing the device) and at all their review appointments.

As shown in Table 2, there was a minor negative change between baseline and follow-up appointments for most of the items evaluated. However, speech, feeling tense and unsatisfactory oral hygiene steeply increased between baseline and 14-day follow-up.

The qualitative data suggested that most of the negative feedback was due to the device
Fig. 2 Mean weight loss of the study participants versus the time points investigated. Error bars represent \pm SD

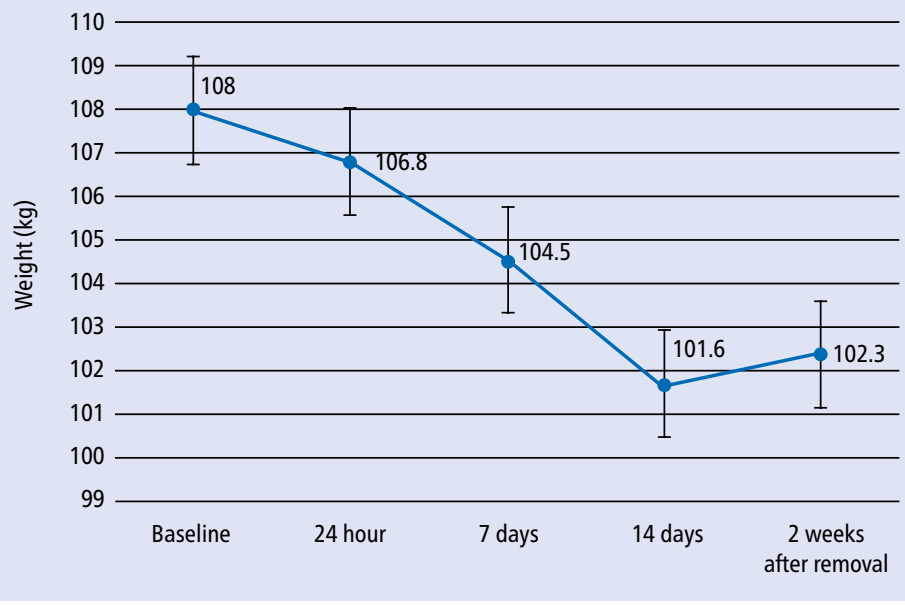

Table 2 Descriptive analysis of the device tolerability

\begin{tabular}{|c|c|c|c|c|c|}
\hline \multirow[t]{2}{*}{ Device tolerability } & Baseline & 24 hours & 7 days & 14 days & $\begin{array}{l}2 \text { weeks } \\
\text { after } \\
\text { removal }\end{array}$ \\
\hline & Mean (SD) & Mean (SD) & Mean (SD) & Mean (SD) & Mean (SD) \\
\hline $\begin{array}{l}\text { Trouble pronouncing } \\
\text { any words }\end{array}$ & $1.29(0.488)$ & $2.86(1.68)$ & $3.00(1.633)$ & $3.00(1.633)$ & $1.00(0)$ \\
\hline Taste changes & $1.57(0.98)$ & $1.43(0.79)$ & $1.86(0.69)$ & $1.86(1.22)$ & $1.00(0)$ \\
\hline Pain & $1.71(0.95)$ & $3.43(1.51)$ & $2.57(1.51)$ & $3.14(1.22)$ & $1.50(.55)$ \\
\hline $\begin{array}{l}\text { Uncomfortable to } \\
\text { drink }\end{array}$ & $1.43(0.79)$ & $2.43(1.51)$ & $1.71(1.11)$ & $2.14(1.46)$ & $1.00(0)$ \\
\hline $\begin{array}{l}\text { Self-conscious } \\
\text { because of device }\end{array}$ & $1.71(0.95)$ & $3.86(1.22)$ & $3.29(1.70)$ & $3.29(1.70)$ & $1.00(0)$ \\
\hline Felt tense & $1.86(1.07)$ & $3.14(1.47)$ & $2.86(1.77)$ & $3.43(1.99)$ & $1.00(0)$ \\
\hline $\begin{array}{l}\text { Oral hygiene is } \\
\text { unsatisfactory }\end{array}$ & $1.57(0.79)$ & $2.57(1.40)$ & $3.14(1.07)$ & $3.43(0.98)$ & $1.83(.99)$ \\
\hline Speech problem & $1.29(0.488)$ & $3.43(1.62)$ & $3.14(1.78)$ & $3.14(1.78)$ & $1.00(0)$ \\
\hline Difficult to relax & $1.43(0.787)$ & $3.57(1.62)$ & $2.71(1.60)$ & $3.14(1.57)$ & $1.00(0)$ \\
\hline Embarrassed & $1.86(1.21)$ & $3.43(1.6)$ & $2.71(1.89$ & $2.86(1.77)$ & $1.00(0)$ \\
\hline Been irritable & $1.71(1.11)$ & $2.89(1.57)$ & $2.14(1.21)$ & $2.57(1.51)$ & $1.00(0)$ \\
\hline $\begin{array}{l}\text { Difficulty doing your } \\
\text { job }\end{array}$ & $1.43(1.13)$ & $2.71(1.60)$ & $2.43(1.81)$ & $2.57(1.51)$ & $1.00(0)$ \\
\hline $\begin{array}{l}\text { Life in general less } \\
\text { satisfying }\end{array}$ & $1.86(1.21)$ & $3.00(1.73)$ & $3.00(1.92)$ & $3.00(1.73)$ & $1.00(0)$ \\
\hline $\begin{array}{l}\text { Totally unable to } \\
\text { function }\end{array}$ & $1.29(.76)$ & $1.57(1.13)$ & $1.57(.976)$ & $1.57(.787)$ & $1.00(0)$ \\
\hline
\end{tabular}


being perceived to be too 'big'. The size of the device was mentioned in 13 different comments overall. The participants linked the size of the device to poor aesthetics rather than discomfort, except one participant who indicated that the device caused friction to her cheek. Two participants indicated that wearing the device affected their work and social life as the device was visible. The most common feedback comments were to 'make it less visible and match it with the colour of teeth'.

Another theme which emerged from the qualitative data was 'happy with outcome. The participants stated the device was tolerable and they would participate in a future study.

Example comments included:

- 'The device was very tolerable'

- 'Device was tolerable and would take part in a future study'

- 'Device was tolerable; however, the design needs to be improved'.

Table 3 illustrates the participants' QoL scores related to physical function, self-esteem, sexual life and public distress. As shown in Table 3, there was a mean increase in QoL related to physical function, self-esteem and sexual life. However, there was no improvement for all the items related to public distress between baseline and 14-day follow-up.

The other themes from the qualitative data were 'motivated to lose weight' and 'give me some savoury food'.
Example comments included:

- 'Became careful of what to eat after losing weight. Avoided sugary foods and foods with carbohydrates'

- 'Started a healthy diet and started working out'

- 'Motivated to continue to lose weight'

- 'Needed something savoury'

- 'Wanted to have a savoury diet like soup during winter'

- 'Would prefer having a mixture of sweet and savoury liquids'.

\section{Discussion}

Obesity is a global pandemic and childhood obesity is widely perceived as one of the most important public health challenges of the twenty-first century. The intraoral device tested is a non-invasive, reversible, attractive and economical alternative to surgical procedures such as bariatric surgery, having little or no morbidity. The device could be helpful for short-term weight loss with a specific goal, such as in obese patients who require knee or hip replacement surgery or in preparation for bariatric surgery, but who will only get their procedure if they lose weight.

Furthermore, it is suggested that the device could break addictive eating habits and show the benefit of reduced weight to avoid future health problems, which may well be serious. The cost of the device and the clinical time to fit and remove the device, which is circa
20 minutes, would be much lower and safer than the main alternative of bariatric surgery. Therefore, it is suggested that many obese people may find this an attractive alternative treatment to achieve successful weight loss.

The intraoral device resulted in a mean weight loss of $6.36(\mathrm{SD}=3.79) \mathrm{kg}$ over a two-week period. All the participants tolerated the device without reporting any serious complications. Jaw wiring has been used previously as a temporary method in the treatment of morbid obesity. Several studies showed that weight losses of 20-30 kg in six months were common with jaw wiring, with a rate of weight loss of around $4.2 \mathrm{~kg} /$ month. ${ }^{18,19,24,25}$ As soon as the wires were removed, weight gain recurred and the majority of the patients rapidly returned to their pre-treatment weight. ${ }^{17,25,26}$ A similar weight lost rate was observed in this study after two weeks, with the participants only having regained around $0.73 \mathrm{~kg}$ at the final review.

During the review appointment on day seven, the magnets were disengaged, allowing the participants to clean their teeth. No participants reported bad breath or gingival soreness. In contrast, several previous studies have shown that jaw wiring resulted in patients having bad breath and developing periodontal diseases. ${ }^{17,24}$ Surprisingly, few participants stated that they 'occasionally' felt that their oral hygiene was unsatisfactory or complained about unsatisfactory oral hygiene post-device removal in this study. This was unexpected as the patients were unable to

\begin{tabular}{|c|c|c|c|c|c|c|c|c|c|c|}
\hline \multirow{2}{*}{ Physical function } & \multicolumn{2}{|c|}{ Baseline } & \multicolumn{2}{|c|}{24 hours } & \multicolumn{2}{|c|}{7 days } & \multicolumn{2}{|c|}{14 days } & \multicolumn{2}{|c|}{$\begin{array}{l}2 \text { weeks after } \\
\text { removal }\end{array}$} \\
\hline & Mean & SD & Mean & SD & Mean & SD & Mean & SD & Mean & SD \\
\hline Because of my weight, I have trouble picking up objects & 4 & 1.2 & 4.00 & 1.000 & 4.00 & 1.155 & 4.00 & 1.000 & 4.50 & 1.225 \\
\hline Because of my weight, I have trouble tying my shoes & 3.86 & 1.2 & 4.00 & 1.155 & 4.29 & 1.113 & 4.29 & 1.113 & 4.67 & 0.816 \\
\hline $\begin{array}{l}\text { Because of my weight, I have difficulty getting up from } \\
\text { chairs }\end{array}$ & 3.86 & 1.5 & 4.14 & 1.069 & 4.29 & 1.113 & 4.43 & 1.134 & 4.50 & 1.225 \\
\hline Because of my weight, I have trouble using stairs & 3.57 & 1.3 & 3.86 & 1.345 & 4.00 & 1.414 & 4.14 & 1.464 & 4.17 & 1.602 \\
\hline $\begin{array}{l}\text { Because of my weight, I have difficulty putting on or taking } \\
\text { off my clothing }\end{array}$ & 4.14 & 0.69 & 4.43 & 0.535 & 4.57 & 0.535 & 4.71 & 0.488 & 4.83 & 0.408 \\
\hline Because of my weight, I have trouble with mobility & 4.14 & 1.1 & 4.14 & 1.464 & 4.43 & 1.134 & 4.29 & 1.113 & 4.33 & 1.211 \\
\hline Because of my weight, I have trouble crossing my legs & 3.39 & 1.5 & 3.43 & 1.618 & 3.57 & 1.618 & 3.71 & 1.380 & 3.67 & 1.633 \\
\hline I feel short of breath with only mild exertion & 3.57 & 1.3 & 3.29 & 0.951 & 3.43 & 1.134 & 3.57 & 1.134 & 3.17 & 1.329 \\
\hline I am troubled by painful or stiff joints & 3.57 & 1.4 & 3.86 & 1.215 & 3.71 & 0.951 & 4.14 & 0.690 & 3.67 & 1.211 \\
\hline My ankles and lower legs are swollen at the end of the day & 2.43 & 1.1 & 4.71 & 0.488 & 4.43 & 0.787 & 4.57 & 0.787 & 4.33 & 1.033 \\
\hline I am worried about my health & 2.43 & 1.1 & 2.57 & 0.976 & 3.00 & 1.414 & 2.71 & 0.951 & 2.00 & 1.095 \\
\hline
\end{tabular}


open their mouth, which restricted them from brushing their tongue and the lingual and palatal aspects of their teeth regularly. Besides, the chlorohexidine-based mouthwash also caused some discolouration to the tongue. Device tolerability dropped at seven days and improved again at 14 days, which is interesting and could possibly be explained by an extended period of adaptation coupled with increasing cumulative weight loss being noted towards the end of the study.

Jaw wiring possesses a high risk of choking if the patients try to eat solid food or if they vomit. ${ }^{17,25,26}$ In this study, patients were instructed to open their mouth using a unique tool during an emergency (should one eventuate) which was not possible with jaw wiring, which required cutting of the wires in an emergency situation. It is reassuring that during this study, no subject felt the need to release the device using the emergency tool provided.

Most patients experienced occasional discomfort due to the device and it was quite severe after 24 hours, with the friction of the device against the cheeks being the main reason. It is reported that pain and discomfort are common complications during active orthodontic treatment, with pain starting within four hours and increasing over the 24 hours. ${ }^{27,28}$ There is also evidence that about $25-45 \%$ of patients still experience pain after seven days of appliances. ${ }^{29,30}$ Both Stewart et al. and Sergl et al. reported that fixed appliances produced a higher intensity of discomfort than removable appliances. ${ }^{31,32}$ In this study, a similar pattern was observed where the pain increased after 24 hours and decreased after one week, which is interesting as no tooth movement was involved.

Another possible explanation is that some participants decided to remove the cheek protectors during the treatment period, causing friction to the cheeks. No participant received any analgesics for pain management during the treatment period, which is commonly seen in orthodontic treatment. ${ }^{33}$

A striking observation is that patients' speech did not improve during the treatment period. The device consists of closedfield magnets (Fig. 1) which lock the jaw, resulting in limited mouth opening whereby a reasonable degree of normal speech with clarity is possible. ${ }^{21}$ A possible explanation could be that the participants found it difficult to talk for as prolonged a period as they are used to and found that their normal speech was limited. However, following device removal, the participants did not experience any ongoing discomfort, tension or speech problems. Several studies reported that after jaw wiring, patients experienced persistent and limited jaw movement, which was not observed in this study. ${ }^{18,34}$

After 24 hours, the participants indicated that they occasionally felt embarrassed, selfconscious and that life, in general, was less satisfying. Nevertheless, all the participants got accustomed to the device during the treatment period and were able to work effectively in their usual employment. Although all participants found the liquid diet monotonous, participants did not complain of hunger, fatigue, light-headedness or palpitations, which are commonly associated with low-calorie regimens. However, the participants indicated that the food was too sugary and suggested that adding savoury foods to the diet regimen, such as savoury drinks or soups, would be helpful. One patient admitted to 'cheating', consuming melted chocolate and fizzy drinks. This was not surprising as studies have shown that obese patients usually have an addictive personality and an impulsivity for sugary food, and suffer from binge-eating disorders. ${ }^{35,36,37}$

Before placing the device, the majority of the participants indicated that being overweight was the main factor that limited their physical functions, such as using stairs, and caused them to have painful or stiff joints. The participants also felt that weight was the main reason for experiencing public distress, such as being discriminated by others and worrying about fitting through aisles and turnstiles. Furthermore, participants also felt that their self-esteem was low and this was due to being overweight. Several studies have found that obesity has a negative effect on the physical aspects of health-related QoL and mental health. ${ }^{38,39,40}$ None of the participants felt their sexual life was impeded due to their weight before and after the trial. After the trial, the participants' QoL improved, although the items related to public distress remained unchanged. A possible explanation is that the participants did not lose a greater amount of weight over the two-week period, although the amount of weight loss was as expected for this degree of dietary restriction. However, after the two weeks of the trial, the participants indicated that losing weight helped them to carry out regular physical functions and motivated them to embark on a weight loss journey and to drop their BMI to a healthy range.
The limitations of this study were its small size of only seven cases and the short timescale of two weeks. In addition, another limitation is the fact that all the subjects were women. Therefore, future studies should involve a larger number of participants and should be balanced for gender in a parallel-controlled trial. The study was conducted for only two weeks and it is suggested that the participants would have lost more weight as seen in jaw wiring or bariatric surgery if the trial was for a longer period. ${ }^{41}$ When questioned, participants mentioned that the device was too bulky and visible, and they could not enjoy social events such as family dinners. Therefore, the inventors have miniaturised the current device into a slimline device which has a polished appearance with better aesthetics. Future studies can examine the acceptability of this smaller slimline device over extended wear periods.

Unlike bariatric surgery or jaw wiring, this device can be re-applied multiple times in the individual patient. The effect of episodic wear on the periodontal support for the anchor teeth needs to be investigated. However, regular hygiene appointments with device release on a regular basis should prevent any adverse consequences. Thus, weight loss can be obtained progressively with episodic applications interspersed with 'rest periods'. Additionally, if the patient regains weight, they can have the device refitted. Furthermore, the device can be applied by a dentist in about 20 minutes after basic training and it is easy to fit and remove.

\section{Conclusion}

Within the limitations of this study, it can be concluded that all the participants tolerated the device for two weeks and showed satisfactory weight loss. QoL was improved, which motivated the subjects to continue on their weight loss journey.

\section{Conflict of interest}

The authors note that, as anticipated by the ethics application, patent protection in the device was sought, with that being done through a new, nonactive, holding company in which the research collaborators had proportionate ownership interests.

\section{Acknowledgements}

The authors wish to acknowledge Jean Legros for her continuous support as the dental assistant and to all study participants for their time and interest in the study. No funding was received for this paper. 


\section{References}

1. Hales C M, Carroll M D, Fryar C D, Ogden C L. Prevalence of obesity among adults and youth: United States, 2015-2016. NCHS Data Brief 2017; 288: 1-8.

2. Ahirwar R, Mondal P R. Prevalence of obesity in India: A systematic review. Diabetes Metab Syndr 2019; 13: 318-321.

3. Kelly T, Yang W, Chen C S, Reynolds K, He J. Globa burden of obesity in 2005 and projections to 2030. Int J Obes (Lond) 2008; 32: 1431-1437.

4. Bray G A. Complications of obesity. Ann Intern Med 1985: 103(6(Pt 2)): 1052-1062.

5. Pi-Sunyer FX. Medical hazards of obesity. Ann Intern Med 1993; 119(7 Pt 2): 655-660.

6. Esfahani S B, Pal S. Obesity, mental health, and sexual dysfunction: A critical review. Health Psychol Open 2018, DOI: 10.1177/2055102918786867.

7. Finer N, Garnett S P, Bruun J M. COVID-19 and obesity. Clin Obes 2020; DOI: 10.1111/cob.12365.

8. Sattar N, McInnes I B, McMurray J J V. Obesity Is a Risk Factor for Severe COVID-19 Infection: Multiple Potential Mechanisms. Circulation 2020; 142: 4-6.

9. Tobias D K, Pan A, Jackson C L et al. Body-mass index and mortality among adults with incident type 2 diabetes. N Engl J Med 2014; 370: 233-244.

10. Torgerson J S, Hauptman J, Boldrin M N, Sjostrom L. XENical in the Prevention of Diabetes in Obese Subjects (XENDOS) study: a randomized study of orlistat as an adjunct to lifestyle changes for the prevention of type 2 diabetes in obese patients. Diabetes Care 2004; 27 : 155-161.

11. Haemmingsson E, Johansson K, Eriksson J, Sundstrom J, Neovius M, Marcus C. Weight loss and dropout during a commercial weight-loss programme including a verylow-calorie diet, a low-calorie diet, or restricted normal food: observational cohort study. Am J Clin Nutr 2012; 96: 953-961.

12. Greenway F L, Bray G A. Combination drugs for treating obesity. Curr Diab Rep 2010; 10: 108-115.

13. Bray G A, Greenway F L. Current and potential drugs for treatment of obesity. Endocr Rev 1999; 20: 805-875.

14. Birkmeyer J D, Finks J F, O'Reilly A et al. Surgical skill and complication rates after bariatric surgery. $N$ Engl J Med 2013; 369: 1434-1442.
15. Jakobsen G S, Smastuen M C, Sandbu R et al. Association of Bariatric Surgery vs Medical Obesity Treatment With Long-term Medical Complications and Obesity-Related Comorbidities. JAMA 2018; 319: 291-301.

16. Malinowski SS. Nutritional and metabolic complications of bariatric surgery. Am J Med Sci 2006 331: $219-225$

17. Castelnuovo-Tedesco P, Buchanan D C, Hall H D. Jaw-wiring for obesity. Gen Hosp Psychiatry 1980; 2: 156-159.

18. Rodgers S, Burnet $R$, Goss $A$ et al. Jaw wiring in treatment of obesity. Lancet 1977; 1: 1221-1222.

19. Goss A N. Management of patients with jaws wired for obesity. A review of 122 cases. Br Dent J 1979; 146: 335-339.

20. Bjorvell H, Hadell K, Jonsson B, Molin C, Rossner S. Long-term effects of jaw fixation in severe obesity. Int J Obes 1984; 8: 79-86.

21. Hall R. Obesity treatment aid (Google Patents). 2003. Available online at https://patents.google.com/ patent/AU9212701A/en (accessed May 2021).

22. Kolotkin R L, Head S, Hamilton M, Tse C K. Assessing Impact of Weight on Quality of Life. Obes Res 1995; 3: 49-56.

23. Kolotkin R L, Head S, Brookhart A. Construct validity of the Impact of Weight on Quality of Life Questionnaire. Obes Res 1997; 5: 434-441.

24. Garrow J S, Gardiner G T. Maintenance of weight loss in obese patients after jaw wiring. Br Med J (Clin Res Ed) 1981; 282: 858-860

25. Kark A E. Jaw wiring. Am J Clin Nutr 1980; 33(2 Suppl): 420-424.

26. Harding P E. Jaw wiring for obesity. Lancet 1980; 1 : $534-535$

27. Hussain A S, Al Toubity M J, Elias W Y. Methodologies in Orthodontic Pain Management: A Review. Open Dent J 2017; 11: 492-497.

28. Marques LS, Paiva S M, Vieira-Andrade R G, Pereira L J, Ramos-Jorge M L. Discomfort associated with fixed orthodontic appliances: determinant factors and influence on quality of life. Dental Press J Orthod 2014; 19: $102-107$
29. Scheurer PA, Firestone A R, Burgin W B. Perception of pain as a result of orthodontic treatment with fixed appliances. Eur J Orthod 1996; 18: 349-357.

30. Bergius M, Berggren U, Kiliaridis S. Experience of pain during an orthodontic procedure. Eur J Oral Sci 2002; 110 92-98.

31. Sergl H G, Klages U, Zentner A. Pain and discomfort during orthodontic treatment: causative factors and effects on compliance. Am J Orthod Dentofacial Orthop 1998; 114: 684-691.

32. Stewart F N, Kerr W J, Taylor P J. Appliance wear: the patient's point of view. Eur J Orthod 1997; 19: 377-382.

33. Patel $S$, McGorray S P, Yezierski R, Fillingim R, Logan $H_{\text {, }}$ Wheeler T T. Effects of analgesics on orthodontic pain. Am J Orthod Dentofacial Orthop 2011; DOI: 10.1016/j. ajodo.2010.07.017.

34. Towers J F. Jaw fixation in massive obesity. In Maxwell J D, Gazet J C, Pilkington P R (eds) Surgical management of obesity. pp 57-66. London: Academic Press, 1980.

35. Lent M R, Swencionis C. Addictive personality and maladaptive eating behaviours in adults seeking bariatric surgery. Eat Behav 2012; 13: 67-70.

36. Schag K, Schonleber J, Teufel M, Zipfel S, Giel K E. Foodrelated impulsivity in obesity and binge eating disorder-a systematic review. Obes Rev 2013: 14: 477-495.

37. Wonderlich S A, Joiner Jr T E, Keel P K, Williamson D A, Crosby R D. Eating disorder diagnoses: empirical approaches to classification. Am Psychol 2007; 62: $167-180$

38. van Vuuren CL, Wachter G G, Veenstra R et al. Associations between overweight and mental health problems among adolescents, and the mediating role of victimization. BMC Public Health 2019; 19: 612.

39. Larsson U, Karlsson J, Sullivan M. Impact of overweight and obesity on health-related quality of life-a Swedish population study. Int J Obes Relat Metab Disord 2002; 26: 417-424

40. Huang I C, Frangakis C, Wu A W. The relationship of excess body weight and health-related quality of life: evidence from a population study in Taiwan. Int $\mathrm{J}$ Obes (Lond) 2006; 30: 1250-1259.

41. Livhits M, Mercado C, Yermilov I et al. Preoperative predictors of weight loss following bariatric surgery: systematic review. Obes Surg 2012; 22: 70-89. 